\title{
Prevalence of Group B Streptococcus serotypes III and V in pregnant women of Rio de Janeiro, Brazil
}

\author{
Georgia Cristina Tavolaro Soares ${ }^{1}$, Daniela Sales Alviano², Gabriela da Silva Santos ${ }^{1}$, \\ Celuta Sales Alviano ${ }^{2}$, Ana Luiza Mattos-Guaraldi ${ }^{3}$, Prescilla Emy Nagao ${ }^{1}$ \\ ${ }^{1}$ Departamento de Biologia Celular, Instituto de Biologia Roberto Alcântara Gomes, \\ Universidade do Estado do Rio de Janeiro, RJ, Brazil. \\ ${ }^{2}$ Departamento de Microbiologia Geral, Instituto de Microbiologia Prof. Paulo de Góes, \\ Universidade Federal do Rio de Janeiro, RJ, Brazil. \\ ${ }^{3}$ Disciplina de Microbiologia e Imunologia, Faculdade de Ciências Médicas, \\ Universidade do Estado do Rio de Janeiro, RJ, Brazil.
}

Submitted: February 27, 2012; Approved: November 13, 2012

\begin{abstract}
GBS serotypes III and V were the most prevalent in pregnant women and exhibited resistance to tetracycline, clindamycin and sulfamethoxazole/trimethoprim. Serotype III showed high sialic acid content and PFGE analysis discerned 33 heterogeneous profiles. Phenotypic and genotypic characterization could be relevant to control GBS infections unaffected by intra-partum chemoprophylaxis.
\end{abstract}

Key words: Group B Streptococcus, antimicrobial susceptibility, sialic acid, PFGE.

Streptococcus agalactiae (Group B Streptococcus, GBS) are the most common cause of serious bacterial infections in newborn and adults with underlying medical disorders. At the time of birth, GBS is vertically transmitted to the neonate, and it is estimated that $50 \%$ or more of newborns exposed to GBS will become colonized if the mother is a carrier (Trappe et al., 2011)

The first-line agent against GBS infection is penicillin, and penicillin resistance among GBS strains has not been reported so far. However, for patients allergic to penicillin, macrolides (e.g., erythromycin) and lincosamides (e.g., clindamycin) are the alternative choices for the treatment of GBS infections. In Brazil, previous studies showed resistance to tetracycline $(21.36 \%)$, erythromycin $(5.83 \%)$ and clindamycin (1.94\%) in cities located in the Southern and Southeast regions (Oliveira et al., 2005).

Although the physical structure of the capsular polysaccharide (CPS) of each GBS serotype is unique, all ten different GBS serotypes (Ia, Ib, II-IX) invariably share a terminal $\alpha$-2,3-linked $N$-acetylneuraminic acid (sialic acid), which is identical to the predominant sialic acid found on human cells. GBS sialic acid enhances their virulence by interfering specifically with opsonic components of the complement system and, represent a key step toward understanding the mechanisms of Sia-dependent virulence at the host-pathogen interface (Weiman et al., 2010). CPS serotyping is the classic method for typing GBS in epidemiological studies. However, phenotypic methods have limited accuracy and often result in some non-typeable isolates. Earlier studies have demonstrated the discriminatory ability and reliability of pulsed-field gel electrophoresis (PFGE) in further differentiation of GBS strains of the same serotype (Rolland et al., 1999).

The purpose of this study was to characterize GBS isolates recovered from pregnant healthy women attended in a public hospital located in the metropolitan area of Rio de Janeiro, Brazil, analyzing genotypic (PFGE) and phenotypic profiles related to serotype, antibiotic susceptibility and sialic acid content.

Thirty-three isolates consecutively isolated from pregnant ( $>25$ weeks of gestation) women were mostly obtained with a swab cultured from the lower vagina. No one had any signs or symptoms of infection at the moment of sampling (unpublished data). Microorganisms were formerly identified as group B by means of a latex-agglutination grouping kit (Streptococcal Grouping Kit, Oxoid,

Send correspondence to P.E. Nagao. Departamento de Biologia Celular, Instituto de Biologia Roberto Alcântara Gomes, Universidade do Estado do Rio de Janeiro, Maracanã, Rio de Janeiro, RJ, Brazil. E-mail: pnagao@pq.cnpq.br. 
São Paulo, Brazil) and serotyped by immunoprecipitation in agarose using new sera produced locally and $\mathrm{HCl}$ antigen extracts from 20-mL broth cultures (Lancefield, 1933). GBS were stored after lyophilisation and recovered in Brain Heart Infusion broth (BHI; Difco). Six Brazilian strains 85147 and 90222 (serotype Ia; oropharynx and urine, respectively), 80340 and 90356 (serotype III; vagina and cerebrospinal fluid), 88641 and 90186 (serotype V; vagina and blood) partially evaluated in some virulence features (Costa et al., 2011) were also included in all assays.

GBS isolates were tested for susceptibility to the following antibiotics by the disk diffusion method considering breakpoints established in M100-S21 CLSI standards (2011): ampicillin $(10 \mu \mathrm{g})$, cefazolin $(30 \mu \mathrm{g})$, clindamycin $(2 \mu \mathrm{g})$, erythromycin $(15 \mu \mathrm{g})$, imipenem $(10 \mu \mathrm{g})$, norfloxacin $(5 \mu \mathrm{g})$, penicillin (10 IU), sulfamethoxazole/trimethoprim $(23.75 \mu \mathrm{g} / 1.25 \mu \mathrm{g})$, tetracycline $(30 \mu \mathrm{g})$, and vancomycin $(30 \mu \mathrm{g})$ (Baltimore Biological Laboratories [BBL], Sparks, MD).

For sialic acid analyses, GBS isolates grown in $100 \mathrm{~mL}$ of BHI for $18 \mathrm{~h}$ at $37^{\circ} \mathrm{C}$ were recovered by centrifugation, washed once in $0.01 \mathrm{M}$ PBS and lyophilised. After determination of the weight of lyophilised cells, the extraction procedures were carried out based on methods previously described by Kamerling and colleagues (Kamerling et al., 1982).

For clonality analyses using PFGE, plugs containing genomic DNA were prepared as described by Rolland and colleagues (Rolland et al., 1999). DNA was digested by SmaI restriction enzyme (Amersham Biosciences do Brasil Ltda, São Paulo, SP) and submitted to PFGE with a program as follows: switch time of 1 to $30 \mathrm{~s}, 23 \mathrm{~h}, 120^{\circ}$ angle, $11.3{ }^{\circ} \mathrm{C}$ and a voltage gradient of $6 \mathrm{~V} / \mathrm{cm}$. Lambda phage concatemers were used as DNA size standards with size range $50-1,000 \mathrm{~kb}$.

The criteria used for definition of the clonality were those suggested by Tenover et al. (1995). DNA banding patterns were analysed for similarity as follows: [i] Genetically indistinguishable if their restriction patterns had the same number of bands and the same apparent size (they were assigned the same capital letter and numerical index); [ii] Closely related to the predominant strain (assigned here as $A_{1}$ pattern) if the PFGE profile differed from the profile $A_{1}$ by changes that resulted in two or three band differences; [iii] Possibly related to the predominant strain if the PFGE profile differed from the predominant $A_{1}$ profile by changes with four to six band differences; [iv] Unrelated to the predominant PFGE pattern if the PFGE pattern differed from the profile $A_{1}$ by changes consistent with seven or more band differences. An unrelated isolate was considered to belong to a different clone and was ascribed a different capital letter. GBS isolates belonging to a specific serotype and displaying identical, closely related, or possibly related PFGE profiles were assigned to the same pattern (Tenover et al., 1995).
In attempt of classifying minor typing pattern variations a supplementary analysis was performed as suggested by Tenover et al. (1997). Therefore, quantitative pairwise comparison of pulsotypes was accomplished by using the Dice coefficient of similarity. A dendrogram was constructed by using the software Bionumerics (Version 2.0 Applied Maths, Kortrijk, Belgium) to provide an image of the genetic relationship between isolates with arithmetic averages with a tolerance coefficient of $1.0 \%$ and optimization of $1.0 \%$. PFGE patterns were defined grouping isolates that showed $>70 \%$ dendrogram identity, as previously used in some GBS strains (Savoia et al., 2008).

The presence of GBS vaginal or rectal carriage was detected in $24.3 \%$ of parturient women observed in our community. The carriage rate found by us was higher when compared with the results described in Santa Catarina (21.6\%) (Pogere et al., 2005), Ribeirão Preto (17.9\%) (Zusman et al., 2006) and São Luis (20.4\%) (Costa et al., 2008) (Fisher's Exact Test, $\mathrm{p}=0.0192, \mathrm{p}=0.005, \mathrm{p}=0.0143$, respectively). However, the present data were similar to those described by other authors in Brazil: Rio de Janeiro (26\%) (Benchetrit et al., 1982) and Campinas (27.6\%) (Nomura et al., 2006).

Among the 39 GBS isolates studied, the serotypes III and $\mathrm{V}$ were the most prevalent and the most resistant to antibiotics in agreement with the results of other authors (Oliveira et al., 2005). Epidemiological analysis, showed that serotype III was the most prevalent with 15 (38.46\%) isolates, followed by 10 (25.64\%) isolates of serotype $\mathrm{V}, 3$ (7.69\%) of serotype II, 4 (10.26\%) of serotype Ia, 2 (5.13\%) of serotype $\mathrm{Ib}$, and $5(12.82 \%)$ non-typeable isolates (Table 1).

The analysis of the antimicrobial susceptibility profiles of 39 GBS isolates revealed colonizing isolates, universally susceptible to ampicillin, cefazolin, erythromycin, imipenem, norfloxacin, penicillin, and vancomycin (Table 1). Conversely, $69.23 \%$ and $7.69 \%$ isolates were resistant to tetracycline and clindamycin, respectively. In another study from Rio de Janeiro, a high prevalence of tetracycline resistance was also observed among clinical isolates from human sources (90.9\%) (Duarte et al., 2005).

A significative number of currently assessed GBS isolates were resistant to sulfamethoxazole/trimethoprim (17.95\%). Frequent resistance to sulfamethoxazole/trimethoprim (53.85\%) was previously reported among GBS isolates in Southern Croatia (Barisiæ et al., 2003), but not in any region of Brazil.

As reported in a previous study from Kuwait, GBS was the third most common cause of urinary tract infection, and the recommended therapy for acute uncomplicated cystitis in adults is sulfamethoxazole/trimethoprim (Sweih et $a l ., 2005)$. Therefore, local epidemiological data are necessary in order to choose appropriate antimicrobial therapy. High level antimicrobial resistance among colonized, pregnant women is likely driven by antibiotic use, coupled with 
Table 1 - Characteristics of the multiple-strain clonal clusters of 39 GBS isolates in Rio de Janeiro

\begin{tabular}{|c|c|c|c|c|c|c|c|c|}
\hline \multirow{3}{*}{ Serotype } & \multicolumn{8}{|c|}{ Number of isolates } \\
\hline & \multirow[t]{2}{*}{ Total } & \multirow[t]{2}{*}{ PFGE patterns } & \multicolumn{3}{|c|}{ Resistance phenotypes } & \multicolumn{3}{|c|}{ Sialic acid content $\mathrm{t}^{\mathrm{a}}$} \\
\hline & & & SMX/TMP & TET & CLIN & $<2$ & $2-4$ & $\geq 4$ \\
\hline Ia & 4 & $A_{1}(2), B_{1}(1), B_{2}(1)$ & $\mathrm{R}(1)$ & $\mathrm{R}(2)$ & $\mathrm{R}(1)$ & 3 & 0 & 1 \\
\hline $\mathrm{Ib}$ & 2 & $\mathrm{~A}_{2}(1), \mathrm{C}_{1}(1)$ & $\mathrm{R}(1)$ & $\mathrm{R}(1)$ & $\mathrm{R}(0)$ & 0 & 1 & 1 \\
\hline II & 3 & $\mathrm{D}_{1}(1), \mathrm{D}_{2}(1), \mathrm{E}(1)$ & $\mathrm{R}(1)$ & $\mathrm{R}(2)$ & $\mathrm{R}(0)$ & 1 & 2 & 0 \\
\hline III & 15 & $\begin{array}{c}\mathrm{F}_{1}(2), \mathrm{F}_{2}(1), \mathrm{G}_{1}(1), \mathrm{H}_{1}(2), \\
\mathrm{I}_{1}(1), \mathrm{J}_{1}(1), \mathrm{K}_{1}(2), \mathrm{L}_{1}(1) \\
\mathrm{M}_{1}(1), \mathrm{N}_{1}(1), \mathrm{N}_{2}(1), \mathrm{O}_{1}(1)\end{array}$ & $\mathrm{R}(2)$ & $\mathrm{R}(12)$ & $\mathrm{R}(1)$ & 3 & 8 & 4 \\
\hline V & 10 & $\begin{array}{c}P_{1}(2), P_{2}(1), Q_{1}(1), R_{1}(1) \\
S_{1}(2), T_{1}(1), U_{1}(1), V_{1}(1)\end{array}$ & $\mathrm{R}(2)$ & $\mathrm{R}(7)$ & $\mathrm{R}(1)$ & 4 & 5 & 1 \\
\hline NT & 5 & $\mathrm{~W}_{1}(1), \mathrm{W}_{2}(1), \mathrm{X}_{1}(1), \mathrm{Y}_{1}(1), \mathrm{Z}_{1}(1)$ & $\mathrm{R}(0)$ & $\mathrm{R}(3)$ & $\mathrm{R}(0)$ & 2 & 3 & 0 \\
\hline
\end{tabular}

SMX/TMP, trimethoprim-sulphamethoxazole; TET, tetracycline; CLIN, clindamycin; NT, non-typeable. ${ }^{\mathrm{a}} \mu \mathrm{g} / \mathrm{mg}$ of cells dry weight.

high transmission probabilities. Our current findings highlight the need for routine susceptibility testing of GBS, particularly in individuals with penicillin allergy, to ensure proper therapy. In Rio de Janeiro, penicillin remains the first choice among drugs for prophylaxis and treatment of early-onset GBS infections in our community.

The distribution of sialic acid levels in the serotypes of GBS tested was classified into three categories: (i) Low sialic acid content of less than $2.0 \mu \mathrm{g} / \mathrm{mg}$ of cell dry weight; (ii) Moderate sialic acid content of more than 2.0 but less than $4.0 \mu \mathrm{g} / \mathrm{mg}$; (iii) High sialic acid content of equal to or more than $4.0 \mu \mathrm{g} / \mathrm{mg}$. All GBS strains exhibited sialic acid content at different levels. Higher $(\geq 4 \mu \mathrm{g} / \mathrm{mg})$ contents were observed among some strains of serotypes Ia, Ib, III and $\mathrm{V}$.

DNA fingerprinting by PFGE revealed identical genomic profiles demonstrating clonality amongst a few number of GBS isolates (Figure 1). Analysis of band patterns of GBS isolates by using both criteria established by Tenover et al. (1995) and Dice coefficient of similarity (Savoia et al., 2008) showed distinct clonal origin for the majority of isolates. However, different results were observed by both methods for some samples.

The criteria proposed by Tenover et al. (1995) that defined genetic events and categories of genetic and epidemiological relatedness allowed the differentiation of GBS isolates into 33 pulsotypes as follows: type Ia and Ib isolates, 3 and 2 patterns, respectively; type II strains, 3 patterns; type III and V, 12 and 8 patterns; non-typeable strains, 5 patterns. No predominant patterns were detected among GBS isolates.

Figure 1 also illustrates the results of analysis of computer-generated dendrogram using the Dice coefficient. Data showed minor typing pattern variations for some strains. Dice coefficient analysis was unable to show various genetic events that occurred in each isolated, which leads to different patterns of bands detected in the Tenover method. A particular pulsotype (B1 and B2) showed Dice

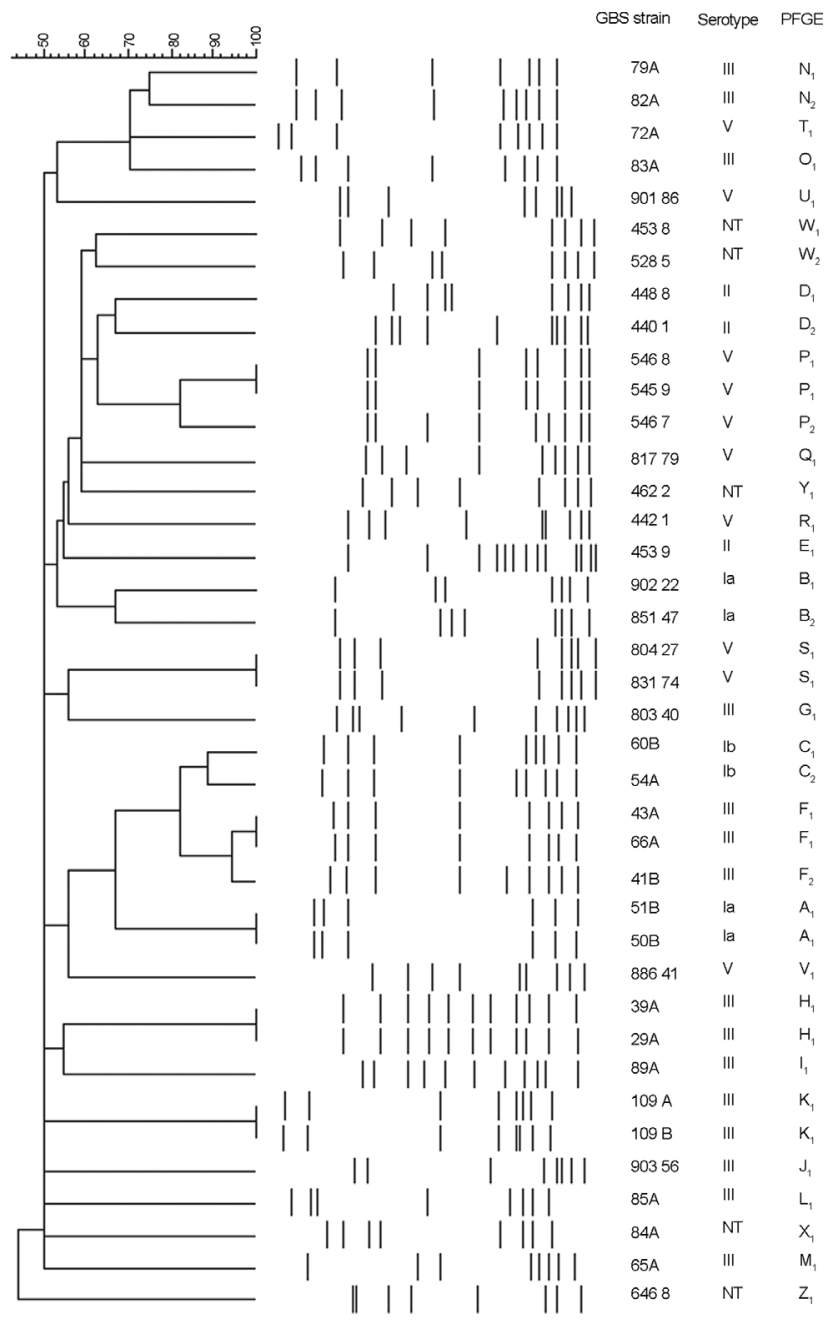

Figure 1 - Phylogenetic analysis of PFGE profiles for GBS isolates. The dendrogram was constructed with PFGE profiles by similarity and clustering analyses using the unweighted-pair group method with arithmetic averages and Dice's coefficient. The levels of genetic similarity in percentages are shown on the left. The strain serotype and codes PFGE subtype are indicated on the right. 
coefficient less than $70 \%$ of similarity. Conversely, different pulsotypes (F1, F2, C1 and C2) showed Dice coefficient with higher than $80 \%$ similarity. Data showed the relevance of using both methods of analysis of PFGE patterns of GBS endemic isolates.

In conclusion, the high degree of genetic heterogeneity of the GBS strains not only among the different polysaccharide-specific serotypes, but within each serotype as well, indicates the endemic nature of GBS infection in our community. However, PFGE-type clonal groups were observed for GBS III, V and Ia serotypes. Moreover, an increased resistance to tetracycline, clindamycin and sulfamethoxazole/trimethoprim was observed among GBS strains isolated from asymptomatic carriers from Rio de Janeiro. Data also emphasize that a simple combined strategy of intrapartum prophylaxis with universal neonatal screening for GBS and an assessment of risk factors for perinatal GBS disease could significantly reduce the rate of lateonset GBS infections in our community.

\section{Acknowledgments}

We thank Mariléa Temperini and Vanessa Figueiredo Albino for their dedicated field and technical support.This work was supported by FAPERJ, CNPq and SR-2/UERJ.

\section{References}

Barisiæ Z, Babiæ-Erceg A, Borziæ E, Zoraniæ V, Kaliterna V, Carev M (2003) Rinary tract infections in South Croatia: aetiology and antimicrobial resistance. Int $\mathrm{J}$ Antimicrob Agents 22:S61-S64.

Benchetrit LC, Fracalanzza SEL, Peregrine H, Camelo AA, Sanches LA (1982) Carriage of Streptococcus agalactiae in women and neonates and distribution of serological types: a study in Brazil. J Clin Microbiol 15:787-790.

Clinical and Laboratory Standard Institute. (2011). Performance Standards for Antimicrobial Susceptibility Testing; 20th Informational Supplement. M100-S20. NCCLS, Wayne, PA.

Costa AL, Lamy FF, Chein MB, Brito LM, Lamy ZC, Andrade KL (2008) Prevalence of colonization by group B Streptococcus in pregnant women from a public maternity of Northwest region of Brazil. Rev Bras Ginecol Obstet 30:274-280.

Costa AF, Pereira CS, Santos GS, Carvalho TM, Hirata JrR, Mattos-Guaraldi AL, Rosa AC, Nagao PE (2011) Group B Streptococcus serotypes III and V induce apoptosis and necrosis of human epithelial A549 cells. Int J Mol Med 27:739-744.

Duarte RS, Bellei BC, Miranda OP, Brito MAVP, Teixeira LM (2005) Distribution of Antimicrobial Resistance and Virulence-Related Genes among Brazilian Group B Streptococci Recovered from Bovine and Human Sources. Antimicrob Agents Chemother 49:97-103.

Kamerling JP, Dorland L, van Halbeek H, Vliegenthart JF, Messer M, Schauer R (1982) Structural studies of 4-O-acetyl-
alpha-N-acetylneuraminyl-(2 goes to 3)-lactose, the main oligosaccharide in echidna milk. Carbohydr Res 1:331-340.

Lancefield RC (1933) A serological differentiation of specific types of bovine hemolytic Streptococci (group B). J Exp Med 59:441-458.

Nomura ML, Júnior RP, Oliveira UM (2006) Selective versus non-selective culture medium for group B Streptococcus detection in pregnancies complicated by preterm labor or preterm-premature rupture of membranes. Braz J Infect Dis 10:247-250

Oliveira ICM, Mattos MC, Areal MFT, Ferreira-Carvalho BT, Figueiredo AMS, Benchetrit LC (2005) Pulsed-field gel electrophoresis of human group B Streptococci isolated in Brazil. J Chemother 17:258-263.

Pogere A, Zoccoli CM, Tobouti NR, Freitas PF, d'Acampora AJ, Zunino JN (2005) Prevalence of group B Streptococcus in pregnant women from a prenatal care center. Rev Bras Ginecol Obstet 27:174-180.

Rolland K, Marois C, Siquier V, Cattier B, Quentin R (1999) Genetic features of Streptococcus agalactiae strains causing severe neonatal infections, as revealed by pulsed field gel electrophoresis and hylB gene analysis. J Clin Microbiol 37:1892-1898.

Savoia D, Gottimer C, Crocilla C, Zucca M (2008) Streptococcus agalactiae in pregnant women: Phenotypic and genotypic characters. J Infect 56:120-125.

Sweih NA, Jamal W, Rotimi VO (2005) Spectrum and antibiotic resistance of uropathogens isolated from hospital and community patients with urinary tract in two large hospitals in Kuwait. Med Princ Pract 14:401-407.

Tenover FC, Arbeit RD, Goering RV, Mickelsen PA, Mrray BE, Persing DH, Swaminathan B (1995) Interpreting chromosomal DNA restriction patterns produced by pulsed-field gel electrophoresis: Criteria for bacterial strain typing. J Clin Microbiol 33:2233-2239.

Tenover FC, Arbeit RD, Goering RV (1997) How to select and interpret molecular strain typing methods for epidemiological studies of bacterial infections: a review for healthcare epidemiologists. Molecular Typing Working Group of the Society for Healthcare Epidemiology of America. Infect Control Hosp Epidemiol 18:426-439.

Trappe KL, Shaffer LE, Stempel LE (2011) Vaginal-perianal compared with vaginal-rectal cultures for detecting group B streptococci during pregnancy. Obstet Gynecol 118:313-317.

Weiman S, Uchiyama S, Lin FY, Chaffin D, Varki A, Nizet V, Lewis AL (2010) O-Acetylation of sialic acid on Group B Streptococcus inhibits neutrophil suppression and virulence. Biochem J 428:163-168.

Zusman AS, Baltimore RS, Fonseca SNS (2006) Prevalence of maternal group B streptococcal colonization and related risk factors in a brazilian population. Braz $\mathrm{J}$ Infect Dis 10:242-246.

All the content of the journal, except where otherwise noted, is licensed under a Creative Commons License CC BY-NC. 